\title{
openheart Genomic ancestry as a predictor of haemodynamic profile in heart failure
}

\author{
Sabrina Bernardez-Pereira, ${ }^{1,2}$ Luciana Gioli-Pereira, ${ }^{2}$ \\ Fabiana G Marcondes-Braga, ${ }^{2}$ Paulo Caleb Junior Lima Santos, ${ }^{2}$ \\ Joceli Mabel Rocha Spina, ${ }^{2}$ Andréa Roseli Vançan Russo Horimoto, ${ }^{2}$ \\ Hadassa Campos Santos, ${ }^{2}$ Fernando Bacal, ${ }^{2}$ Fábio Fernandes, ${ }^{2}$ \\ Alfredo Jose Mansur, ${ }^{2}$ Ricardo Pietrobon, ${ }^{3}$ José Eduardo Krieger, ${ }^{2}$ \\ Evandro Tinoco Mesquita, ${ }^{1}$ Alexandre Costa Pereira ${ }^{2}$
}

To cite: Bernardez-Pereira S, Gioli-Pereira L,

Marcondes-Braga F G, et al. Genomic ancestry as a predictor of haemodynamic profile in heart failure. Open Heart 2016:3:e000434. doi:10.1136/openhrt-2016000434

- Additional material is available. To view please visit the journal (http://dx.doi.org/ 10.1136/openhrt-2016000434).

Received 14 March 2016 Revised 5 June 2016 Accepted 28 June 2016

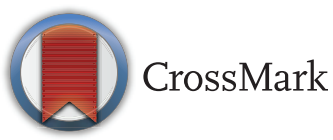

${ }^{1}$ Fluminense Federal University, Niteroi, Rio de Janeiro, Brazil

${ }^{2}$ Heart Institute, University of São Paulo Medical School, Sao Paulo, Brazil

${ }^{3}$ University of California, San Francisco, California, USA

Correspondence to Dr Sabrina Bernardez-Pereira; bernardezsabrina@gmail.com

\section{ABSTRACT}

Objective: The aim of this study is to assess the association between genetic ancestry, self-declared race and haemodynamic parameters in patients with chronic heart failure (HF).

Methods: Observational, cross-sectional study. Eligible participants were aged between 18 and 80 years; ejection fraction was $\leq 50 \%$. Patients underwent genetic analysis of ancestry informative markers, echocardiography and impedance cardiography (ICG). Race was determined by self-classification into two groups: white and non-white. Genomic ancestry was estimated using a panel of 101348 polymorphic markers and three continental reference populations (European, African and Native American).

Results: Our study included 362 patients with HF between August 2012 and August 2014. 123 patients with HF declared themselves as white and 234 patients declared themselves as non-white. No statistically significant differences were found regarding the ICG parameters according to self-declared race. The Amerindian ancestry was positively correlated with systolic time ratio $(r=0.109, p<0.05)$. The thoracic fluid content index $(r=0.124$. $p<0.05)$, E wave peak $(r=0.127 . p<0.05)$ and $E / e^{\prime}$ ratio $(r=0.197 . p<0.01)$ were correlated positively with African ancestry. In multiple linear regression, African ancestry remained associated with the E/e $\mathrm{e}^{\prime}$ ratio, even after adjustment to risk factors.

Conclusions: The African genetic ancestry was associated with worse parameters of diastolic function; the Amerindian ancestry correlated with a worse pattern of ventricular contractility, while self-declared colour was not helpful to infer haemodynamic profiles in HF.

Trials registration number: NTC02043431.

\section{INTRODUCTION}

Heart failure (HF) is a major cause of morbidity and mortality in the world, with a major impact on health systems. ${ }^{1}$ Despite the available therapies, this syndrome is now the

\section{KEY QUESTIONS}

What is already known about this subject?

- Incidence and prognosis in heart failure (HF) differ across ethnic groups. Mortality and hospitalisation rates for $\mathrm{HF}$ are higher among African-Americans than among their white counterparts.

What does this study add?

- The African genetic ancestry was associated with worse parameters of diastolic function in patients with HF while the Amerindian ancestry was associated with higher values of systolic time ratio and raises the hypothesis of a possible explanation for the differences in prognosis between the ethnic groups.

How might this impact on clinical practice?

- Genetic analysis, once their cost is reduced to a cost-effective level, should be incorporated into clinical practice to define high-risk groups and guide treatment in HF.

leading cause of hospitalisation in persons aged over 65 years and is a major contributor to increased costs of healthcare. ${ }^{12}$

Previous studies have also shown that the incidence and prognosis of HF differ across ethnic groups. Mortality and hospitalisation rates for $\mathrm{HF}$ are higher among AfricanAmericans than among their white counterparts. ${ }^{34}$

Disparity in the prevalence and outcomes of HF among blacks and whites has been attributed to a wide range of factors. Among them are racial/ethnic differences in the prevalence of underlying diseases, quality and availability of healthcare and disparities in socioeconomic factors. ${ }^{5} 6$ However, isolated ethnic differences in socioeconomic indicators cannot fully explain the high mortality among black patients. ${ }^{7}$ This finding has reinforced the idea that part of the 
ethnic differences in cardiovascular events is mediated by genetic factors that might determine disease severity and response to therapy in $\mathrm{HF}^{9}$

Pathophysiological mechanisms that are aetiologically linked to the development of heart diseases tend to be disproportionately prevalent in selected racial/ethnic groups. African descents has been related to salt sensitivity, excessive renin-angiotensin system-dependent target-organ injury, endothelium-dependent and endothelium-independent vascular abnormal responses. These evidences suggest that this racial group can present a worse haemodynamics in HF leading to worse outcomes. ${ }^{10}$

Furthermore, studies have shown that, compared to European descendants, African-American patients have important differences in the frequencies of functionally significant alleles encoding important components of neurohormonal signalling cells in $\mathrm{HF}^{11-15}$ Results are still controversial, and a clear association between different HF endophenotypes, including haemodynamic pattern and genetic ancestry, has not been previously explored. Therefore, the objective of this study is to assess the association of genetic ancestry with noninvasive haemodynamic parameters in patients with chronic HF and reduced ejection fraction from a multiethnic population.

\section{MATERIAL AND METHODS}

GENIUS-HF is an observational, prospective cohort, single-centre study and it is still ongoing. The individuals invited to our study were consecutively selected from the Heart Institute-Clinical Hospital- University of São Paulo Medical School (InCor-HCFMUSP). Its methodology has been previously published. ${ }^{16}$ This analysis is a cross-sectional evaluation of the association between haemodynamic profile and genomic ancestry conducted between August 2012 and August 2014.

\section{Eligibility criteria}

The eligibility criteria are the same as in the GENIUS-HF study. We included patients between 18 and 80 years of age, with a history and physical examination compatible with $\mathrm{HF}$, left ventricular ejection fraction (LVEF) $\leq 50 \%$ documented in transthoracic Doppler echocardiography in the past year. Exclusion criteria were advanced dementia or any other mental or psychiatric disorders that would limit the collection of information; patients who refused the provision of informed consent form and, for this analysis, patients who have not been evaluated by impedance cardiography (ICG) for any reason.

\section{Ethical aspects}

The Ethics Committee for Medical Research in Human of the Clinical Hospital of the School of Medicine, University of São Paulo approved the study protocol (Protocol number 0398/04-SDC 2368/03/162) and all individuals signed an informed consent form. This study was registered at Clinical Trials.gov under number NTC02043431.

\section{Study procedures}

Once eligible, patients underwent clinical evaluation and laboratory testing, including transthoracic Doppler echocardiography, ICG and blood collection. Patients were interviewed at a medical appointment for knowledge about medical history, symptoms, medication use, HF aetiology and socioeconomic measures.

Race was self-identified according to the Brazilian Institute of Geography and Statistics (IBGE) for race classification: white, black, 'pardo' (an intermediate colour category-brown), yellow or indigenous. ${ }^{17}$ For the purposes of this analysis, we categorised them into two groups: white (self-declared white) and non-white (other self-declared race). ${ }^{17}$

\section{Echocardiogram and haemodynamic evaluation}

Haemodynamic data were obtained in a non-invasive manner using the CardioScreen 2000 haemodynamic measurement system (Medis-Ilmenau, Germany). The parameters, definitions and normal values for each variable can be found in online supplementary material 1 . Doppler echocardiography was performed simultaneously with the ICG in an Acuson Sequoia S512 system (Siemens, Pennsylvania, USA). The following parameters were assessed: left ventricular (LV) cavity diameters and left atrial volume index; thickness of the posterior wall and interventricular septum; LVEF, the peak Doppler velocities of early (E) and late diastolic flow (A), the deceleration time and the $\mathrm{E} / \mathrm{A}$ ratio. The ratio of mitral velocity to early diastolic velocity of the mitral annulus $\left(\mathrm{E} / \mathrm{E}^{\prime}\right)$ was also measured.

\section{Genetic molecular analysis}

\section{Genotyping data}

Individuals from the GENIUS-HF study were genotyped on the Axiom assay from genomic DNA on the AxiomTM Genome-Wide InCor_BB Array, a custom chip that genotypes $\sim 800000$ single nucleotide polymorphisms (SNPs). To analyse genetic ancestry, we used the shared SNPs between the reference populations and GENIUS-HF samples, totalling 101348 common SNPs.

\section{Data cleaning}

We applied two filters for SNP cleaning: (1) missing genotype rate per marker, where no markers containing genotype missing rates above $10 \%$ were identified; (2) Hardy-Weinberg equilibrium (HWE), where 10201 markers were excluded from our sample based on the HWE test $(p \leq 0.0001)$. We did not apply a filter for minor allele frequency $(\mathrm{MAF}<0.01)$ since markers in the genotyping platform were rare.

Three exploratory tools were applied to evaluate sample quality: (1) missing genotype rate, where no samples demonstrated more than $10 \%$ of missing 
genotypes; (2) Identity by descent (IBD) analysis, where we identified 13 pairs of duplicate individuals and (3) a sex analysis check, where we verified any incompatibility between informed and genotypically evaluated gender. All data cleaning procedures were performed using the PLINK package. ${ }^{18}$

\section{Genomic ancestry}

The evaluation of genomic ancestry was conducted using the Admixture program. ${ }^{19}$ Admixture is a software tool for maximum likelihood estimation of individual ancestries from multilocus SNP genotype data sets. Since the contributions of different ancestral genomes have previously been described by our group, as well as others, we used a supervised approach for ancestry determination. ${ }^{20}$ Parameters were set at 200 bootstrap replicates (default) and three populations were assumed for the analysis.

This analysis was performed using all 101348 common autosomal SNPs between the study and reference populations. ${ }^{21}$ We assumed as reference ancestral populations all individuals from the Human Genome Diversity Project (HGDP): ${ }^{22}$ Pima, Maya as Amerindians and from the HapMap project: ${ }^{23}$ Africans-YRI (Yoruba in Ibadan, Nigeria), LWK (Luhya in Webuye, Kenya), ASW (Americans of African Ancestry in SW, USA); European-CEU (Utah Residents (CEPH) with Northern and Western European ancestry) and TSI (Tuscan in Italia). ${ }^{24} 25$

\section{Data collection system}

Study data were collected and managed using the electronic data capture application REDCap (Research Electronic Data Capture), hosted at the Hospital das Clinicas of the School of Medicine, University of São Paulo. REDCap is an application designed to have security compliant with common requirements. ${ }^{26}$

\section{Statistical analysis}

We used the $\chi^{2}$ or Fisher's exact tests to compare categorical variables between white and non-white groups. The parameters of ICG and the variables of ancestry were normally distributed, except for the variable $\mathrm{O} / \mathrm{C}$ ratio. This variable was therefore log-transformed. Genetic ancestry variables were used as continuous variables. Correlation between numerical variables was analysed using Pearson's correlation coefficient. Student's t-test was used to determine differences between the means of ICG and echocardiographic variables by gender and self-declared race (white and non-white). Multiple linear regression analysis using as the dependent variable lateral $\mathrm{E} / \mathrm{e}^{\prime}$ ratio was built in two models (table 5). The first model included the African ancestry while the second model used the non-white selfreported race. Both models also estimated the interaction between age and chronic renal failure and the variables associated with the lateral $\mathrm{E} / \mathrm{e}^{\prime}$ ratio in the univariate analysis (see online supplementary material 2 ).
Owing to the high penetration of Chagas disease in this study, which could confuse the interpretation of results, we performed a sensitivity analysis on the sample of patients without Chagas disease (see online supplementary material 3). Additionally, we used a generalised linear model with a Gaussian family distribution to estimate predicted mean of lateral $\mathrm{E} / \mathrm{e}^{\prime}$ with $95 \%$ CIs for each $10 \%$ of genomic ancestry and for self-reported race (white and non-white). Estimates were adjusted for the same variables of the model. The tests, although multiple, are correlated and our sample size is not powered for conservative multiple testing correction approaches.

Statistical analyses were conducted using the SPSS V.16.0 software (SPSS, Illinois, USA) and statistical significance was set at the 0.05 level.

\section{RESULTS}

We included 500 patients between August 2012 and August 2014, all meeting the eligibility criteria and consenting to participate in this study. Of these, 2 withdrew informed consent, 364 underwent ICG and 317 transthoracic echocardiography. Data from 2 participants of 364 undergoing ICG on admission visit had incomplete information and were therefore discarded from our analysis. Of the 362 remaining patients considered for this subanalysis, genetic evaluations from 14 patients were excluded for not meeting our quality criteria. Our overall study workflow is depicted in figure 1 .

\section{Self-declared race, clinical characteristics and treatment}

The clinical characteristics and treatment according to the self-declared ethnicity (white and non-white) are in table 1.

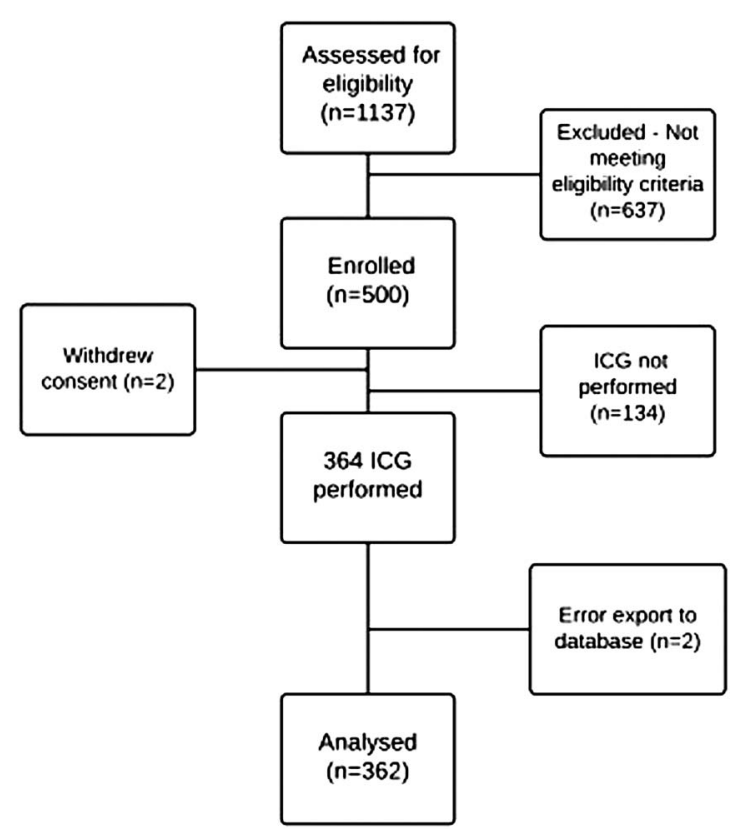

Figure 1 GENIUS-HF study flow chart. ICG, impedance cardiography. 
Table 1 Clinical features and treatment according to the self-declared race

\begin{tabular}{|c|c|c|c|}
\hline Variables & White $(n=123)$ & Non-white $(n=234)$ & p Value \\
\hline Age (years)-mean $\pm S D$ & $57.5 \pm 13.6$ & $55.0 \pm 12.2$ & 0.076 \\
\hline Gender (male)-n (\%) & $79(64.2)$ & $158(67.5)$ & 0.611 \\
\hline Arterial hypertension (\%) & $72(58.5)$ & $156(66.7)$ & 0.16 \\
\hline Dyslipidaemia (\%) & $62(50.4)$ & $121(51.7)$ & 0.902 \\
\hline Prior myocardial infarction (\%) & $39(31.7)$ & $58(24.9)$ & 0.212 \\
\hline Atrial fibrillation (\%) & $18(14.6)$ & 27 (11.5) & 0.503 \\
\hline Current smoking (\%) & $12(9.8)$ & $15(6.4)$ & 0.355 \\
\hline \multicolumn{4}{|l|}{ Aetiology (\%) } \\
\hline Hypertensive & $19(15.4)$ & $55(23.5)$ & \\
\hline Ischaemic & $38(30.9)$ & $47(20.1)$ & \\
\hline Chagas disease & $16(13.0)$ & $55(23.5)$ & 0.024 \\
\hline LVEF (\%)-mean+SD & $32.8 \pm 8.3$ & $31.3 \pm 8.7$ & 0.132 \\
\hline Functional III/IV-n (\%) & $28(22.8)$ & $40(17.1)$ & 0.248 \\
\hline$\beta$-blocker-n (\%) & $120(97.6)$ & $226(96.6)$ & 0.852 \\
\hline ACEi/ARB-n (\%) & $68(55.3)$ & $144(61.8)$ & 0.281 \\
\hline Education level $\leq$ elementary school-n (\%) & $74(60.7)$ & $183(78.2)$ & 0.001 \\
\hline Familiar income $(\mathrm{R} \$)$-mean $\pm \mathrm{SD}$ & $1792.0 \pm 1327.2$ & $1619.2 \pm 1095.2$ & 0.194 \\
\hline
\end{tabular}

Self-declared 'yellow' and native patients represented a small portion ( 5 patients) of the overall studied population.

Ischaemic aetiology was significantly more prevalent in the self-declared white group $(30.9 \%$ vs $20.1 \%$, $\mathrm{p}=0.032$ ), whereas Chagas disease was more prevalent in self-declared non-white patients $(23.5 \%$ vs $13.0 \%$, $\mathrm{p}=0.026)$. Patients in the self-declared non-white group more commonly had an educational level only up to elementary school $(78.2 \%$ vs $60.7 \%, \mathrm{p}=0.001)$. Age, gender, comorbidities, body mass index, functional class, LVEF, socioeconomic status and use of ACE inhibitors/ angiotensin-receptor blocker or $\beta$-blockers did not significantly differ by self-declared race.

\section{Comparison between self-declared race and genomic ancestry}

When evaluating our study sample, the distribution of average European, African and Amerindian ancestry was, respectively, $61 \%, 29 \%$ and $6 \%$. Self-reported ethnicity was represented as follows: 123 white patients (34.5\%); 179 brown patients (50.1\%); 55 black patients (15.4\%); 5 patients classified as other $(1.4 \%)$.

Patients self-reported as white presented an average of $80 \%$ European ancestry, 12\% of African ancestry and $8 \%$ of Amerindian ancestry. Among self-declared brown patients, genetic ancestry was 58\% European, 31\% African and $11 \%$ Amerindian. Self-declared black patients had on average 32\% European ancestry, $61 \%$ African ancestry and 7\% Amerindian ancestry.

In figure 2, we observed that self-identified white patients have a higher percentage of European descent when compared to self-declared non-white patients. Self-identified non-white patients showed higher African descent compared to self-identified white patients. The occurrence of Amerindian ancestry was below $40 \%$ in groups (white and non-white).

As expected, mean genetic ancestry (European, African and Amerindian) demonstrated statistically

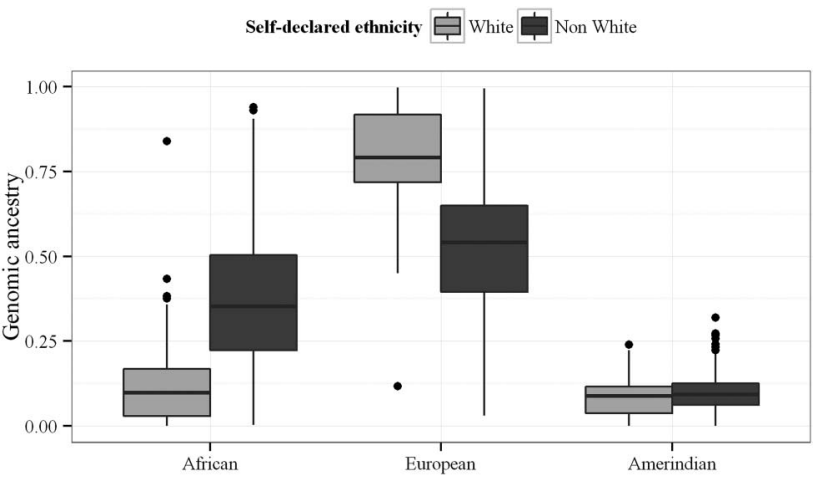

Figure 2 Comparison of genomic ancestry with self-declared race. 
Table 2 Comparison of self-declared race with the genetic ancestry

\begin{tabular}{|c|c|c|c|c|}
\hline \multirow[b]{2}{*}{ Genomic ancestry } & \multicolumn{3}{|c|}{ Self-declared race } & \multirow[b]{2}{*}{ p Value } \\
\hline & White & Brown & Black & \\
\hline European & $0.797 \pm 0.146$ & $0.584 \pm 0.160$ & $0.319 \pm 0.167$ & $<0.001$ \\
\hline African & $0.120 \pm 0.120$ & $0.309 \pm 0.155$ & $0.612 \pm 0.185$ & $<0.001$ \\
\hline Amerindian & $0.084 \pm 0.059$ & $0.107 \pm 0.052$ & $0.069 \pm 0.046$ & $<0.001$ \\
\hline
\end{tabular}

significant differences in relation to self-declared race (white, brown and black) $(\mathrm{p}<0.001$; table 2$)$.

\section{Haemodynamic parameters and self-declared race}

The adjusted association between ICG parameters and self-declared race did not demonstrate any statistically significant differences between the white and non-white groups (table 3 ).

Comparing adjusted mean values from the Doppler echocardiography evaluation between white and nonwhite groups, the averages of the interventricular septum $(9.4 \mathrm{~mm}$ vs $8,8 \mathrm{~mm}, \mathrm{p}=0.004)$, posterior wall of the left ventricle $(9.5 \mathrm{~mm}$ vs $9,2 \mathrm{~mm}, \mathrm{p}=0.046)$, LV mass index $\left(149 \mathrm{~g} / \mathrm{m}^{2}\right.$ vs $\left.136 \mathrm{~g} / \mathrm{m}^{2}, \mathrm{p}=0.023\right)$ and the medial $\mathrm{E} / \mathrm{e}^{\prime}$ ratio ( 9 vs $7, \mathrm{p}=0.016$ ) were higher for the nonwhite group (table 3 ).

\section{Haemodynamic parameters and genomic ancestry}

Correlation between ICG parameters and Doppler echocardiography with the European, Amerindian and African ancestry showed a weak positive correlation to the systolic time ratio with Amerindian ancestry $(\mathrm{r}=0.109, \mathrm{p}<0.05)$. The thoracic fluid content $(\mathrm{r}=0.124$, $\mathrm{p}<0.05)$, the wave $\mathrm{E}$ peak $(\mathrm{r}=0.127, \mathrm{p}<0.05)$ and the medial $\mathrm{E} / \mathrm{e}^{\prime}$ ratio $(\mathrm{r}=0.142, \mathrm{p}<0.05)$ and lateral $\mathrm{E} / \mathrm{e}^{\prime}$ ratio $(\mathrm{r}=0.197, \mathrm{p}<0.01)$ correlated positively with African ancestry, while European ancestry was negatively correlated with the wave $\mathrm{E}$ peak $(\mathrm{r}=-0.118, \mathrm{p}<0.05)$ and medial $\mathrm{E} / \mathrm{e}^{\prime}$ ratio $(\mathrm{r}=-0.120, \mathrm{p}<0.05)$ (table 4$)$.

Table 5 shows the results of multiple linear regression analysis.

In model 1 , African ancestry $(\mathrm{p}=0.005)$, chronic renal failure $(\mathrm{p}=0.005)$, mean arterial pressure

Table 3 ICG and echocardiographic parameters in HF according to the self-declared ethnicity

\begin{tabular}{|c|c|c|c|}
\hline & White $(n=123)$ & Non-white $(n=234)$ & p Value* \\
\hline \multicolumn{4}{|l|}{ ICG parameters } \\
\hline $\mathrm{MAP}(\mathrm{mm} \mathrm{Hg})$ & $88 \pm 15$ & $91 \pm 16$ & 0.166 \\
\hline $\mathrm{PP}(\mathrm{mm} \mathrm{Hg})$ & $45 \pm 16$ & $47 \pm 17$ & 0.148 \\
\hline $\mathrm{HR}(\mathrm{L} / \mathrm{min})$ & $70 \pm 15$ & $67 \pm 14$ & 0.157 \\
\hline $\mathrm{Cl}\left(\mathrm{L} / \mathrm{min} / \mathrm{m}^{2}\right)$ & $2.6 \pm 0.6$ & $2.4 \pm 0.6$ & 0.150 \\
\hline $\mathrm{SI}\left(\mathrm{mL} / \mathrm{m}^{2}\right)$ & $38 \pm 11$ & $37 \pm 10$ & 0.492 \\
\hline SVRI (dyn.s.cm $\left.{ }^{-5} \cdot \mathrm{m}^{2}\right)$ & $2743 \pm 863$ & $2998 \pm 958$ & 0.055 \\
\hline STR & $0.33 \pm 0.17$ & $0.35 \pm 0.19$ & 0.391 \\
\hline TFCl $\left(1 / K O h m / m^{2}\right)$ & $19 \pm 5$ & $20 \pm 4$ & 0.668 \\
\hline \multicolumn{4}{|c|}{ Echocardiographic parameters } \\
\hline LA vol index (vol/m²) & $40 \pm 16$ & $41 \pm 20$ & 0.652 \\
\hline IVS (mm) & $8.8 \pm 2$ & $9.4 \pm 2$ & 0.004 \\
\hline LVPW (mm) & $9.2 \pm 2$ & $9.5 \pm 2$ & 0.046 \\
\hline LVDD (mm) & $64 \pm 10$ & $65 \pm 9$ & 0.450 \\
\hline LVSD (mm) & $52 \pm 10$ & $51 \pm 10$ & 0.869 \\
\hline LVEF (\%) & $39 \pm 10$ & $40 \pm 9$ & 0.468 \\
\hline LV mass index $\left(\mathrm{g} / \mathrm{m}^{2}\right)$ & $136 \pm 46$ & $149 \pm 47$ & 0.023 \\
\hline Wave E peak & $0.7 \pm 0.2$ & $0.7 \pm 0.3$ & 0.423 \\
\hline Wave A peak & $0.6 \pm 0.2$ & $0.6 \pm 0.2$ & 0.625 \\
\hline Medial E/e' ratio & $7 \pm 3$ & $9 \pm 4$ & 0.016 \\
\hline Lateral E/e' ratio & $6 \pm 3$ & $7 \pm 3$ & 0.100 \\
\hline SPAP (mm Hg) & $31 \pm 10$ & $31 \pm 10$ & 0.854 \\
\hline
\end{tabular}

Bold values are statistically significant $(p<0.05)$.

Values expressed as mean \pm SD.

${ }^{*} \mathrm{p}$ Values adjusted for age, sex and aetiologies.

$\mathrm{Cl}$, cardiac index; HF, heart failure; HR, heart rate; ICG, impedance cardiography; IVS, interventricular septal thicknesses; LA vol index, Left atrial volume index; LCWI, left cardiac work index; LV, left ventricular; LVDD, left ventricular diastolic diameter; LVEF, left ventricular ejection fraction; LVPW, left ventricular posterior wall thicknesses; LVSD, left ventricular systolic diameter; MAP, median arterial pressure; PP, pressure pulse; SI, stroke index; SPAP, systolic pulmonary artery pressure; STR, systolic time ratio; SVRI, systemic vascular resistance index; TFCi, thoracic fluid content index. 
Table 4 Correlation of ICG and echocardiographic parameters with genetic ancestry

\begin{tabular}{|c|c|c|c|}
\hline ICG parameters & European & Amerindian & African \\
\hline $\mathrm{MAP}(\mathrm{mm} \mathrm{Hg})$ & -0.61 & -0.061 & 0.081 \\
\hline $\mathrm{PP}(\mathrm{mm} \mathrm{Hg})$ & -0.041 & -0.002 & 0.043 \\
\hline $\mathrm{HR}(\mathrm{L} / \mathrm{min})$ & 0.070 & 0.033 & -0.082 \\
\hline $\mathrm{Cl}\left(\mathrm{L} / \mathrm{min} / \mathrm{m}^{2}\right)$ & -0.003 & -0.032 & 0.013 \\
\hline $\mathrm{SI}\left(\mathrm{mL} / \mathrm{m}^{2}\right)$ & -0.029 & -0.072 & 0.052 \\
\hline SVRI (dyn.s. $\left.\mathrm{cm}^{-5} \cdot \mathrm{m}^{2}\right)$ & -0.044 & 0.007 & 0.043 \\
\hline STR & 0.011 & $0.109 *$ & -0.045 \\
\hline TFCI $\left(1 / \mathrm{KOhm} / \mathrm{m}^{2}\right)$ & -0.104 & -0.058 & $0.124^{\star}$ \\
\hline \multicolumn{4}{|c|}{ Echocardiographic parameters } \\
\hline LA vol index $\left(\mathrm{vol} / \mathrm{m}^{2}\right)$ & 0.034 & -0.026 & -0.027 \\
\hline IVS (mm) & -0.061 & -0.015 & 0.066 \\
\hline LVPW (mm) & -0.041 & -0.045 & 0.056 \\
\hline LVDD (mm) & 0.040 & -0.033 & -0.031 \\
\hline LVSD (mm) & 0.049 & -0.047 & -0.037 \\
\hline LVEF (\%) & -0.044 & 0.077 & 0.023 \\
\hline LV mass index $\left(\mathrm{g} / \mathrm{m}^{2}\right)$ & -0.022 & -0.064 & 0.041 \\
\hline Wave E peak & $-0.118^{\star}$ & -0.021 & $0.127^{\star}$ \\
\hline Wave A peak & 0.022 & 0.023 & -0.029 \\
\hline Medial E/e' ratio & $-0.120^{*}$ & -0.067 & $0.142^{\star}$ \\
\hline Lateral E/e' ratio & -0.173 & -0.070 & $0.197 \dagger$ \\
\hline SPAP $(\mathrm{mm} \mathrm{Hg})$ & 0.052 & -0.106 & -0.022 \\
\hline
\end{tabular}

Bold values are statistically significant.

${ }^{*}$ Correlation significant at the level of $p<0.05$.

tCorrelation significant at the level of $p<0.01$.

$\mathrm{CI}$, cardiac index; HR, heart rate; ICG, impedance cardiography;

IVS, interventricular septal thicknesses; LA vol index, Left atrial

volume index; LCWI, left cardiac work index; LV, left ventricular;

LVDD, left ventricular diastolic diameter; LVEF, left ventricular

ejection fraction; LVPW, left ventricular posterior wall thicknesses;

LVSD, left ventricular systolic diameter; MAP, median arterial

pressure; PP, pressure pulse; SI, stroke index; SPAP, systolic

pulmonary artery pressure; STR, systolic time ratio; SVRI, systemic

vascular resistance index; TFCi, thoracic fluid content index. $(\mathrm{p}=0.001)$, functional class $(\mathrm{p}=0.002)$ and LVEF ( $\mathrm{p}=0.001$ ) were associated with measures of the lateral $\mathrm{E} /$ $\mathrm{e}^{\prime}$ ratio $\left(\mathrm{R}^{2}=18.3 \%\right.$; $\left.\mathrm{AIC}=1461.5\right)$. Model 2 presents the chronic renal failure $(\mathrm{p}=0.008)$, mean arterial pressure $(p<0.001)$, functional class $(p=0.004)$ and LVEF ( $\mathrm{p}=0.001)$ associated with the lateral $\mathrm{E} / \mathrm{e}^{\prime}$ ratio $\left(\mathrm{R}^{2}=16.5 \%\right.$; $\left.\mathrm{AIC}=14678\right)$. The self-declared race was not associated with this variable $(\mathrm{p}=0.165)$.

A positive association between the $\mathrm{E} / \mathrm{e}^{\prime}$ lateral ratio and African ancestry remained positive even after performing a sensitivity analysis where (1) patients with Chagas disease were excluded (see online supplementary material 3) or (2) disease duration and use of diuretics were included in the models.

Estimations of lateral $\mathrm{E} / \mathrm{e}^{\prime}$ for differences in the African ancestry of $10 \%$ and in self-declared race are found in online supplementary material 4 .

Finally, the higher the African ancestry, the higher lateral $\mathrm{E} / \mathrm{e}^{\prime}$. The opposite being true among those of European ancestry, as demonstrated by the association between the mean of lateral $\mathrm{E} / \mathrm{e}^{\prime}$ by quintiles of genomic ancestry (figure 3).

\section{DISCUSSION}

To the best of our knowledge, this is the first study reporting genomic ancestry and haemodynamic pattern in patients with HF. We found that although selfreported race was associated with genetic ancestry, there was a considerable range which ancestry levels would characterise a given self-reported race disagreement between them in a considerable percentage of patients. In addition, the results of this article showed the

Table 5 Multiple linear regression of medial $\mathrm{E} / \mathrm{e}^{\prime}$ ratio as the dependent variable

\begin{tabular}{|c|c|c|c|}
\hline & $\boldsymbol{\beta}$ & $\mathrm{Cl} 95 \%$ & p Value \\
\hline \multicolumn{4}{|l|}{ Model 1. variables } \\
\hline Age (10 years) & -0.08 & -0.38 to 0.22 & 0.595 \\
\hline Chronic renal failure & 5.503 & 1.687 to 9.319 & 0.005 \\
\hline Gender & -0.37 & -1.096 to 0.356 & 0.317 \\
\hline MAP $(1 \mathrm{~mm} \mathrm{Hg})$ & 0.038 & 0.016 to 0.06 & 0.001 \\
\hline NYHA FC (III/IV) & 1.413 & 0.52 to 2306 & 0.002 \\
\hline LV mass index $\left(1 \mathrm{~kg} / \mathrm{m}^{2}\right)$ & 0.004 & -0.003 to 0.012 & 0.263 \\
\hline Ejection fraction (\%) & -0.064 & -0.103 to -0.026 & 0.001 \\
\hline African ancestry (10\%) & 0.225 & 0.069 to 0.380 & 0.005 \\
\hline Interaction: age (10 years) and chronic renal failure & -0.79 & -1.41 to -0.17 & 0.013 \\
\hline \multicolumn{4}{|l|}{ Model 2. variables } \\
\hline Age (10 years) & -0.16 & -0.46 to 0.13 & 0.284 \\
\hline Chronic renal failure & 5.266 & 1.403 to 9.128 & 0.008 \\
\hline Gender & -0.621 & -1.331 to 0.089 & 0.086 \\
\hline MAP (1 mm Hg) & 0.04 & 0.018 to 0.063 & $<0.001$ \\
\hline NYHA FC (III/IV) & 1.345 & 0.443 to 2.246 & 0.004 \\
\hline LV mass index $\left(1 \mathrm{~kg} / \mathrm{m}^{2}\right)$ & 0.005 & -0.003 to 0.012 & 0.227 \\
\hline Ejection fraction (\%) & -0.065 & -0.104 to -0.026 & 0.001 \\
\hline Self-declared race (non-white) & 0.498 & -0.206 to 1.203 & 0.165 \\
\hline Interaction: age (10 years) and chronic renal failure & -0.74 & -1.37 to -0.11 & 0.021 \\
\hline
\end{tabular}

Bold values are statistically significant $(p<0.05)$.

LV, left ventricle; MAP, mean arterial pressure; NYHA FC, New York Heart Association Functional Class. 

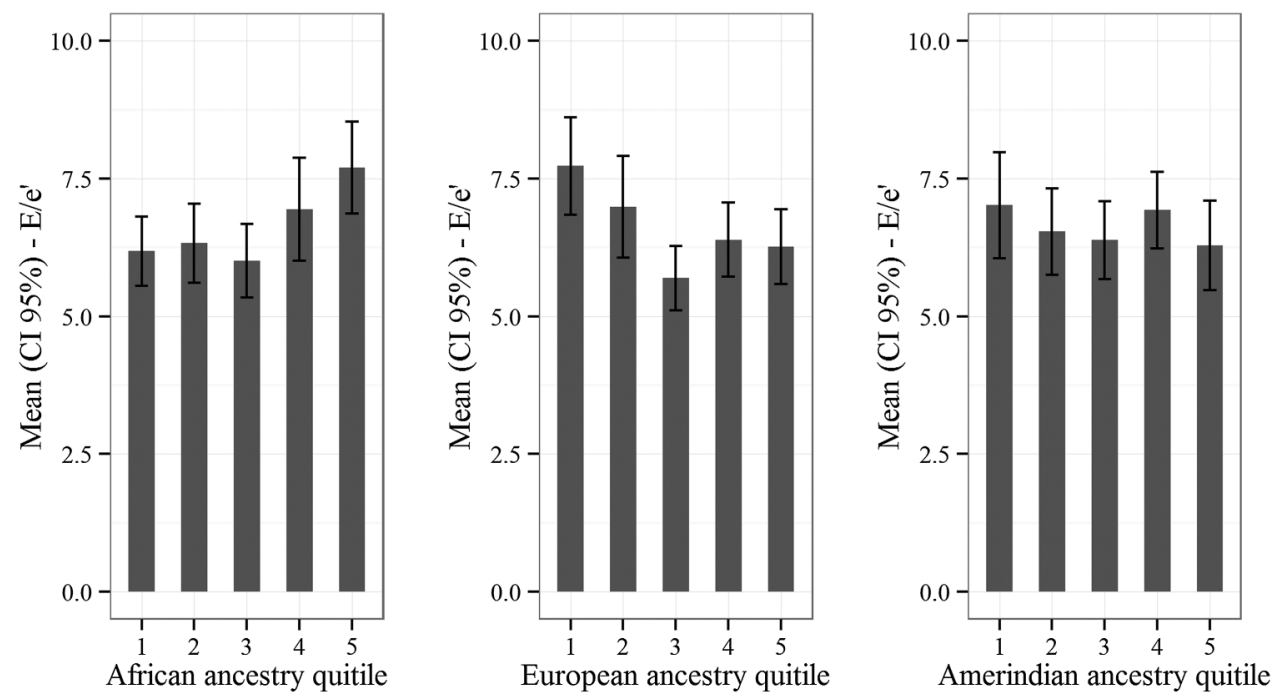

Figure 3 Lateral E/e' ratio and genomic ancestry.

presence of a positive association between peak $\mathrm{E}$ wave, septal and lateral $\mathrm{E} / \mathrm{e} 0$ ratio and thoracic fluid content with African ancestry while the contractility parameter, systolic time ratio, was positively correlated with ancestry Amerindian. Finally, African ancestry showed a positive relationship with the lateral $\mathrm{E} / \mathrm{e}^{\prime}$ ratio, which did not occur for the self-declared non-white.

\section{Genetic ancestry and self-declared race}

The Brazilian population was constituted by an extensive miscegenation among three different ancestral origins: Amerindians, Europeans and Africans. ${ }^{27}$ Our present study demonstrated a predominance of European ancestry in the entire study population. Previous data are in agreement with our findings. ${ }^{19} 2427$

In this study, self-declared race is associated with genetic ancestry. However, our data suggest a higher reliability of the use of genetic ancestry in clinical studies, especially in a mixed population. Feldman et al argue that confusing self-declared race and ancestry can be potentially devastating to the practice of medicine. In one of our arms, we have used a categorical variable (white, brown and black), while the other has been quantified using a continuous variable indicating genetic ancestry (European, Amerindian and African). ${ }^{28}$

\section{Amerindian ancestry and ventricular contractility}

We found a weak positive correlation between the systolic time ratio and Amerindian ancestry. This ICG parameter is derived from the relationship between the pre-ejection and LV ejection time. The higher the systolic time ratio, the worse the ventricular contractility. This ICG parameter has shown to have great potential to be a reliable monitoring method of ventricular function in chronic HF.

Thompson $e t a l^{29}$ demonstrated that systolic time ratio was able to distinguish between HF with preserved function and systolic dysfunction HF. Changes in the cardiac index and systolic time ratio were highly correlated with changes in LVEF. ${ }^{30}$ Furthermore, systolic time ratio proved to be a reliable prognostic parameter in patients with HF. Brain natriuretic peptide (BNP) and systolic time ratio proved to be the strongest future event predictors related to HF. Patients with BNP $>100 \mathrm{pg} / \mathrm{mL}$ and systolic time ratio $>0.45^{\mathrm{sec}-1}$ had a free-events survival rate significantly smaller. ${ }^{31}$

To the best of our knowledge, the only study evaluating genetic ancestry in another sample population of patients with $\mathrm{HF}$ in Brazil demonstrated that the Amerindian ancestry was associated with greater survival rates compared to African and European ancestries, which contradicts our findings. ${ }^{32}$ Once the Amerindian ancestry correlated positively with systolic time ratio and the higher the values of this parameter, the worse the ventricular function, we would expect a worse prognosis. Therefore, new studies in the field are required to have a better understanding of the role of genetic ancestry in the occurrence of events in patients with HF.

\section{African ancestry and diastolic function}

We found that the greater the degree of African ancestry, the higher the indices of the $\mathrm{E} / \mathrm{e}^{\prime}$ ratio. These findings remained significant even after adjustment. However, when a similar model was run for the association with the self-declared non-black race, the association was no longer significant.

A worse diastolic LV performance was observed in African-Caribbean hypertensive patients (expressed by the high values of the $\mathrm{E} / \mathrm{e}^{\prime}$ ratio and lower values $\mathrm{E}$ ' wave) included in the Anglo-Scandinavian Cardiac Outcomes Trial (ASCOT) when compared to their white European counterparts. The role of African-Caribbean ethnicity in diastolic function persisted even after adjustment for potential confounders such as age and LV mass, leading researchers to hypothesise the existence of 
an intrinsic difference in diastolic LV performance between the two racial/ethnic groups. However, this previous analysis did not include the time at diagnosis of hypertension as well as the difference in antihypertensive treatment between the groups. ${ }^{33}$

Russo et al observed, after adjustment for age and sex, that blacks and Hispanics had worse diastolic function when compared to whites. Hispanics showed lower E/A ratio transmitral velocity, lower $\mathrm{E}$ ' wave and higher $\mathrm{E} / \mathrm{e}^{\prime}$ ratio in relation to whites, while blacks had a significantly lower wave velocity medial E' and a tendency to higher $\mathrm{E} / \mathrm{e}^{\prime}$. After adjustment for cardiovascular risk factors and other potential confounders, the authors found no significant differences in the parameters of diastolic function between the three ethnic-racial groups. These findings suggest that most of the observed differences were secondary to significantly imbalanced risk factors, rather than factors intrinsic to ethnicity or race. $^{34}$

Patients in the GENIUS-HF cohort are currently being followed every 6 months for a 3-year follow-up, which will allow us to present new information about prognosis and genetic ancestry.

\section{Limitations}

Despite its novel findings, our study has limitations. First, it is an observational study and thus is not free of selection bias and confounding. Owing to this possibility, some actions were taken as a multivariate analysis to control for confounding factors.

Another issue that could be a limitation in our study is the number of patients who were not evaluated by ICG. Most of the 134 patients did not perform the ICG analysis due to unavailability of the equipment during the enrolment phase of the study. Less than $10 \%$ of all patients undergoing ICG analysis presented factors limiting the examination. Thus, we believe that the selection bias was not of a magnitude that could affect our main results and conclusions.

Regarding statistical analysis, the tests, although multiple, are correlated and our sample size is not powered for conservative multiple testing correction approaches.

Finally, external validity can also be compromised in this study since the genetic ancestry data may vary between regions.

\section{CONCLUSION}

The African genetic ancestry was associated with worse parameters of diastolic function in patients with HF, while the Amerindian ancestry was associated with higher values of systolic time ratio and raises the hypothesis of a possible explanation for the differences in prognosis between the ethnic groups. Additionally, the results of this study emphasise the need to be cautious when using self-declared race as a representative of ancestry or by extrapolating the results of a mixed population to another.
Acknowledgements The authors thank Rafael Muniz Miranda da Silva for his assistance in data management and Lucas Petri Damiani for his statistical modelling assistance.

Contributors SB-P conceived of the GENIUS-HF substudy, designed the data collection tools, contributed to protocol development, monitored the data collection, acquired and analysed the data, and designed the draft. LG-P designed the data collection tools, monitored the data collection, contribute to protocol development and revised the draft paper; FGM-B designed the data collection tools, contribute to protocol development, acquired the data and revised the draft paper. PCJLS provided statistical expertise, analysed the data and revised the draft paper. JMRS planned and managed the data collection and acquired the data. ARVRH provided expertise in genomic analysis and revised the draft paper. HCS provided expertise in genomic analysis and revised the draft paper. FB contributed to protocol development and revised the draft paper. FF contributed to protocol development and revised the draft paper. AJM contribute to protocol development and revised the draft paper. RP provided statistical expertise, analysed the data and revised the draft paper. JEK analysed the data and revised the draft paper. ETM conceived of the substudy; analysed the data and revised the draft paper. AdCP conceived of the GENIUS-HF study, analysed the data and drafted and revised the paper. All authors contributed to refinement of the study protocol and approved the final manuscript.

Funding Fundação Coordenação de Aperfeiçoamento de Pessoal de Nível Superior (CAPES), n11/2009, Brazil. Fundação de Amparo à Pesquisa do Estado de São Paulo (FAPESP)—n 17368-0/2013, Brazil. PROADI-SUS

Program, Brazil Health Ministry, Brazil. This study had no relationship with the industry.

\section{Competing interests None declared.}

Ethics approval The Ethics Committee for Medical Research in Human of the Clinical Hospital of the School of Medicine, University of São Paulo approved the study protocol (Protocol number 0398/04-SDC 2368/03/162).

Provenance and peer review Not commissioned; externally peer reviewed.

Data sharing statement No additional data are available.

Open Access This is an Open Access article distributed in accordance with the Creative Commons Attribution Non Commercial (CC BY-NC 4.0) license, which permits others to distribute, remix, adapt, build upon this work noncommercially, and license their derivative works on different terms, provided the original work is properly cited and the use is non-commercial. See: http:// creativecommons.org/licenses/by-nc/4.0/

\section{REFERENCES}

1. Gheorghiade M, Vaduganathan M, Fonarow GC, et al. Rehospitalization for heart failure: problems and perspectives. J Am Coll Cardiol 2013;61:391-403.

2. Joffe SW, Dewolf M, Shih J, et al. Trends in the medical management of patients with heart failure. J Clin Med Res 2013;5:194-204.

3. Alexander M, Grumbach K, Selby J, et al. Hospitalization for congestive heart failure. Explaining racial differences. JAMA 1995;274:1037-42.

4. Alexander M, Grumbach K, Remy L, et al. Congestive heart failure hospitalizations and survival in California: patterns according to race/ ethnicity. Am Heart J 1999;137:919-27.

5. Hebbar S. Racial differences in the outcome of left ventricular dysfunction. N Engl J Med 1999;341:288.

6. Harjai KJ, Nunez E, Shah M, et al. Does racial bias exist in the medical management of heart failure? Clin Cardiol 2002;25:479-83.

7. Nazroo JY. The structuring of ethnic inequalities in health: economic position, racial discrimination, and racism. Am J Public Health 2003;93:277-84.

8. Rathore SS, Foody JM, Wang Y, et al. Race, quality of care, and outcomes of elderly patients hospitalized with heart failure. JAMA 2003;289:2517-24.

9. Charron $P$, Komajda $M$. Are we ready for pharmacogenomics in heart failure? Eur J Pharmacol 2001;417:1-9.

10. Flack JM, Nasser SA, Levy PD. Therapy of hypertension in African-Americans. Am J Cardiovasc Drugs 2011;11:83-92. 
11. Taylor MR, Sun AY, Davis G, et al. Race, common genetic variation, and therapeutic response disparities in heart failure. JACC Heart Fail 2014;2:561-72.

12. Kurnik D, Li C, Sofowora GG, et al. Beta-1-adrenoceptor geneti variants and ethnicity independently affect response to beta-blockade. Pharmacogenet Genomics 2008;18:895-902.

13. Xie HG, Dishy V, Sofowora G, et al. Arg389Gly beta 1-adrenoceptor polymorphism varies in frequency among different ethnic groups but does not alter response in vivo. Pharmacogenetics 2001;11:191-7.

14. Yancy CW, Fowler MB, Colucci WS, et al, U.S. Carvedilol Heart Failure Study Group. Race and the response to adrenergic blockade with carvedilol in patients with chronic heart failure. $N$ Engl $J$ Med 2001;344:1358-65

15. Pereira SB, Velloso MW, Chermont S, et al. $\beta$-adrenergic receptor polymorphisms in susceptibility, response to treatment and prognosis in heart failure: implication of ethnicity. Mol Med Rep 2013;7:259-65.

16. Gioli-Pereira L, Bernardez-Pereira S, Goulart Marcondes-Braga F et al. Genetic and ElectroNic medical records to predict oUtcomeS in Heart Failure patients (GENIUS-HF) - design and rationale. BMC Cardiovasc Disord 2014;14:32.

17. IBGE IBdGeE-. Censo Demográfico 2000-Características Gerais da População. http://www.ibge.gov.br/home/estatistica/ populacao/censo2000/default_populacao.shtm 2000:1-178.

18. Purcell S, Neale B, Todd-Brown K, et al. PLINK: a tool set for whole-genome association and population-based linkage analyses. Am J Hum Genet 2007;89:559-75.

19. Alexander DH, Novembre J, Lange K. Fast model-based estimation of ancestry in unrelated individuals. Genome research 2009;19:1655-64.

20. Cardena MM, Ribeiro-Dos-Santos A, Santos S, et al. Assessment of the relationship between self-declared ethnicity, mitochondrial haplogroups and genomic ancestry in Brazilian individuals. PLOS ONE 2013:8:e62005.

21. Ginsburg GS, Shah SH, McCarthy JJ. Taking cardiovascular genetic association studies to the next level. J Am Coll Cardiol 2007:50:930-2.

22. SHG C. Human Genome Diversity Project (cited 15 July 2014) http://www.hagsc.org/hgdp/
23. International HapMap Project (cited 15 July 2014) http://hapmap. ncbi.nlm.nih.gov/index.html.en

24. Lima-Costa MF, Rodrigues LC, Barreto ML, et al. Epigen-Brazil group. Genomic ancestry and ethnoracial self-classification based on 5,871 community-dwelling Brazilians (The Epigen Initiative). Sci Rep 2015;5:9812.

25. Kehdy FS, Gouveia MH, Machado M, et al, Brazilian EPIGEN Project Consortium. Origin and dynamics of admixture in Brazilians and its effect on the pattern of deleterious mutations. Proc Natl Acad Sci U S A 2015;112:8696-701.

26. Harris PA, Taylor R, Thielke R, et al. Research electronic data capture (REDCap) - a metadata-driven methodology and workflow process for providing translational research informatics support. $J$ Biomed Inform 2009;42:377-81.

27. Pena SD, Di Pietro G, Fuchshuber-Moraes M, et al. The genomic ancestry of individuals from different geographical regions of Brazil is more uniform than expected. PLOS ONE 2011;6:e17063.

28. Feldman MW, Lewontin RC, King MC. Race: a genetic melting-pot. Nature 2003;424:374.

29. Thompson B, Drazner MH, Dries DL, et al. Systolic time ratio by impedance cardiography to distinguish preserved vs impaired left ventricular systolic function in heart failure. Congest Heart Fail 2008;14:261-5.

30. Parrott CW, Burnham KM, Quale C, et al. Comparison of changes in ejection fraction to changes in impedance cardiography cardiac index and systolic time ratio. Congest Heart Fail 2004;10:11-13.

31. Castellanos LR, Bhalla V, Isakson S, et al. B-type natriuretic peptide and impedance cardiography at the time of routine echocardiography predict subsequent heart failure events. $J$ Card Fail 2009;15:41-7.

32. Cardena MM, Ribeiro-dos-Santos A, Santos S, et al. Amerindian genetic ancestry is associated with higher survival rates compared to African and European ancestry in Brazilian patients with heart failure. Int J Cardiol 2014:176:527-8

33. Sharp A, Tapp R, Francis DP, et al. Ethnicity and left ventricular diastolic function in hypertension an ASCOT (Anglo-Scandinavian Cardiac Outcomes Trial) substudy. J Am Coll Cardiol 2008;52:1015-21.

34. Russo C, Jin Z, Homma S, et al. Race/ethnic disparities in left ventricular diastolic function in a triethnic community cohort. Am Heart J 2010;160:152-8. 